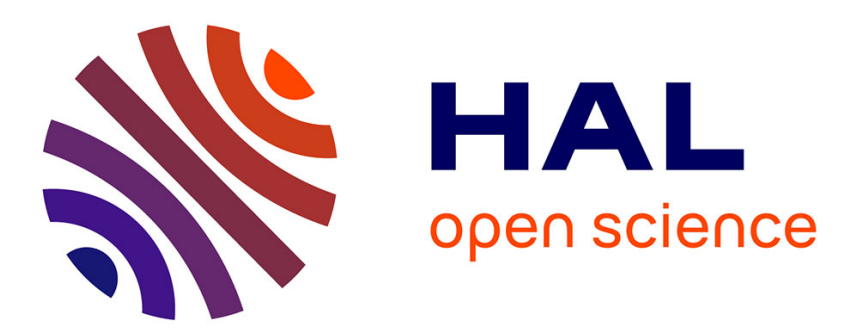

\title{
Can phase shift analysis above the inelastic threshold provide unambiguous results?
}

\author{
J. Bystricky, F. Lehar, P. Winternitz
}

\section{To cite this version:}

J. Bystricky, F. Lehar, P. Winternitz. Can phase shift analysis above the inelastic threshold provide unambiguous results?. Journal de Physique Lettres, 1979, 40 (6), pp.117-118. 10.1051/jphyslet:01979004006011700 . jpa-00231585

\section{HAL Id: jpa-00231585 https://hal.science/jpa-00231585}

Submitted on 1 Jan 1979

HAL is a multi-disciplinary open access archive for the deposit and dissemination of scientific research documents, whether they are published or not. The documents may come from teaching and research institutions in France or abroad, or from public or private research centers.
L'archive ouverte pluridisciplinaire HAL, est destinée au dépôt et à la diffusion de documents scientifiques de niveau recherche, publiés ou non, émanant des établissements d'enseignement et de recherche français ou étrangers, des laboratoires publics ou privés. 


\title{
LE JOURNAL DE PHYSIQUE-LETTRES
}

\section{Can phase shift analysis above the inelastic threshold provide unambiguous results?}

\author{
J. Bystricky, F. Lehar and P. Winternitz $\left(^{*}\right)$ \\ Département de Physique des Particules Elémentaires, CEN-Saclay, 91190 Gif sur Yvette, France
}

(Reçu le 1er décembre 1978, accepté le 19 janvier 1979)

\begin{abstract}
Résumé. - On montre que l'ambiguïté continue présente dans la reconstruction des amplitudes de diffusion à partir des données, due à l'inobservabilité d'une phase globale se réduit à une ambiguïté discrète pour l'analyse en ondes partielles standard et est complètement levée pour l'analyse en ondes partielles modifiée.
\end{abstract}

\begin{abstract}
It is shown that the continuous ambiguity in the reconstruction of scattering amplitudes from data, due to the unobservability of an overall phase, is restricted to a discrete ambiguity in standard partial wave analysis and is removed completely in modified partial wave analysis.
\end{abstract}

It is well known that above the inelastic threshold continuous ambiguities will exist in the direct reconstruction of scattering amplitudes from data [1] and that discrete ambiguities will exist in standard partial wave analysis [2]. It is equally well known that in the actual practice of phase shift analysis for e.g. NN scattering, unique sets of phase shifts are obtained well above the threshold of pion production, as long as the experimental errors are sufficiently small [3].

The purpose of this article is to point out that the disappearance of the ambiguities is due to the actual procedure adopted in phase shift analysis (for brevity and clarity we restrict ourselves to the two-body scattering of spinless particles).

Indeed, consider the reaction $1+2 \rightarrow 3+4$ above the inelastic threshold. The only measurable quantities are the differential and total cross-sections :

$$
\sigma(\theta)=|f(\theta)|^{2}, \quad \sigma_{\mathrm{T}}=\frac{4 \pi}{k} \operatorname{Im} f(0)
$$

We write the usual partial wave expansion of the scattering amplitude (for a fixed energy $k$ ) :

$$
f(\theta)=\sum_{l=0}^{\infty}(2 l+1) a_{l} P_{l}(\cos \theta)
$$

and allow the phase shifts $\delta_{l}$ in the expression

$$
a_{l}=\left(\mathrm{e}^{2 i \delta_{l}}-1\right) / 2 i k
$$

(*) Permanent address : Centre de Recherches Mathématiques, Université de Montréal, Montréal, Québec, Canada. to be complex (their imaginary parts must be positive). Obviously, if we replace $f(\theta)$ by

$$
\tilde{f}(\theta)=f(\theta) \exp i \varphi(\theta),
$$

where $\varphi(\theta)$ is real and satisfies $\varphi(0)=0$, equations (1) remain satisfied. The partial amplitudes $a_{l}$ and phase shifts $\delta_{l}$ will change continuously as we change $\varphi(\theta)$.

The expansion (2) introduces a discrete infinity of phase shifts. In practice two types of partial wave analysis (p.w.a.) are performed, standard and modified. Let us consider them separately.

1. Standard partial wave analysis. - We assume that $a_{l}(k)=0$ for $l \geqslant L+1$, where $L$ is some chosen integer. Thus, $f(\theta)$ is artificially constrained to be a polynomial of order $L$ in $z=\cos \theta$. The question now is : can we replace $f(\theta)$ by another polynomial $\widetilde{f}(\theta)$ of the same order in such a manner that $\widetilde{f}(\theta)$ fits the same data (1) ? The answer is well known [3], namely that a discrete ambiguity group of the order $2^{L+1}$ exists. Thus, the requirement that $f(\theta)$ be a polynomial of order $L$ restricts the continuous ambiguity of $f(\theta)$ to a set of discrete ones.

2. Modified partial wave analysis. - We assume that $a_{l}(k)=A_{l}(k)$ for $l \geqslant L+1$, where $L$ is again a fixed integer and the higher partial waves $A_{l}(k) \neq 0$ are calculated on the basis of some model for the long range part of the interaction involved (m.p.w.a. is typical for studies of nucleon scattering where the quantities $A_{l}(k)$ are provided by the one pion exchange approximation). The question now is : can the function 
$f(\theta)$ be replaced by another function $\tilde{f}(\theta)$ which also describes the data (1) and which has the same tail

$$
\sum_{l=L+1}^{\infty}(2 l+1) A_{l}(k) P_{l}(z)
$$

in its partial wave expansion? To answer this we define

$$
\begin{aligned}
\Delta(\theta)=f(\theta)-\widetilde{f}(\theta)=f(\theta) & \left(1-\mathrm{e}^{i \varphi(\theta)}\right)= \\
& =\sum_{l=0}^{L}(2 l+1) \Delta_{l} P_{l}(z)
\end{aligned}
$$

where $\Delta_{l} \equiv a_{l}-\tilde{a}_{l}$ for $l=0,1, \ldots, L$. The conditions (1) now imply

$$
2 \operatorname{Re}\left[f^{*}(\theta) \Delta(\theta)\right]=|\Delta(\theta)|^{2} \quad \operatorname{Im} \Delta(0)=0 .
$$

The crucial observation is that $f(\theta)$ on the left hand side of the first of equations (4) is an infinite series, whereas $\Delta(\theta)$ is a finite sum. The equation thus represents an infinite set of homogeneous contraints on the $(2 L+2)$ unknown $\operatorname{Im} \Delta_{l}$ and $\operatorname{Re} \Delta_{l}$. Expanding the quantities in (4) we obtain after some manipulations

$$
\begin{gathered}
\sum_{k=0}^{L} \sum_{l=2 L+n-k}^{2 L+n+k}(2 l+1)(2 k+1)(l k 00 \mid 2 L+n 0)^{2}\left\{\operatorname{Re} A_{l} \operatorname{Re} \Delta_{k}+\operatorname{Im} A_{l} \operatorname{Im} \Delta_{k}\right\}=0 \quad n=1,2 \ldots \\
\sum_{l=\max (0, m-L)}^{L} \sum_{k=|l-m|}^{\min (l+m, L)}(2 l+1)(2 k+1)(l k 00 \mid m 0)^{2}\left[\Delta_{l} \Delta_{k}^{*}-2 \operatorname{Re} a_{l}^{*} \Delta_{k}\right]= \\
=2 \sum_{l=L+1}^{L+m} \sum_{k=|l-m|}^{L}(2 l+1)(2 k+1)(l k 00 \mid m 0)^{2} \operatorname{Re}\left(A_{l}^{*} \Delta_{k}\right) \quad 0 \leqslant m \leqslant 2 L \\
\sum_{l=0}^{L}(2 l+1) \operatorname{Im} \Delta_{l}=0 .
\end{gathered}
$$

We have analysed the system (5) and come to the following conclusions. In general no solution exists, except for the trivial one $\Delta_{k}=$ for $k=1, \ldots, L$. For some very special choices of the calculated amplitudes $A_{l}$ solutions may exist. Thus, for standard p.w.a. we have $A_{l}=0$ and $(5 a)$ is satisfied trivially and system (5b) and $(5 c)$ has some solutions. If $\operatorname{Im} A_{l}=0$ for all $l$, then no nontrivial solutions exist. If $\operatorname{Re} A_{l}=0$ for all $l$, then the ambiguity $\delta_{l} \rightarrow-\delta_{l}$ exists.

To summarize : in the direct reconstruction of the scattering amplitudes for spinless particules above the inelastic threshold a continuous ambiguity exists, due to the nonobservability of the overall phase of $f(\theta)$. This ambiguity is restricted to a discrete one in standard partial wave analysis and is in general removed completely in modified partial wave analysis.

Similar methods can be used to analyse ambiguities in the reconstruction of scattering amplitudes for the scattering of particles with spin, in particular pion nucleon scattering [3-5]. Again the overall phase is unobservable and cannot be obtained in a direct reconstruction. A partial wave analysis reduces the ambiguity at most to a discrete one and modified partial wave analysis removes this ambiguity completely [6]. The degree of ambiguity in standard p.w.a. decreases with respect to the spinless case, since the number of linearly independent experiments grows like the square of the number of amplitudes.

The role of a model for the higher waves in p.w.a. is thus a crucial one. It not only reduces the number of parameters in the amplitude to a manageable size and stabilizes the convergence, but also removes the ambiguity in the overall phase of the scattering amplitudes. In particular the one pion exchange amplitudes in nucleon scattering do remove all ambiguities in the NN partial wave analysis.

\section{References}

[1] Atkinson, D., Johnson, P. W. and Warnock, R. L., Commun. Math. Phys. 28 (1972) 133, 33 (1973) 221.

Atkinson, D., Mahoux, G. and Yndurain, F. J., Nucl. Phys. B 54 (1973) 263, B 66 (1973) 429.

[2] Gersten, A., Nucl. Phys. B 12 (1965) 537.

[3] Bystricky, J. and Lehar, F., Proc. IV Intern. Symp. Pol. Phen. in Nucl. Reactions, Zürich, 1975, p. 458 (Birkhäuser Verlag, Basel) 1976.
[4] Berends, F. A. and Ruijsenaars, S. N. M., Nucl. Phys. B 56 (1973) $507,525$.

[5] Brunet, R. C., Gauthier, L. and Winternitz, P., Phys. Rev. D 13 (1976) 1350.

[6] Bystricky, J., Lehar, F. and Winternitz, P., Publication CRM-763, Montreal, 1978. 